\title{
Introduction to Electronic Marketing Minitrack
}

\author{
Bruce D. Weinberg \\ Department of Marketing \\ Isenberg School of Management \\ University of Massachusetts, Amherst \\ 121 Presidents Drive \\ Amherst, MA 01003 \\ Weinberg@Isenberg.umass.edu
}

\author{
Lenita M. Davis \\ Department of Marketing \\ College of Business \\ University of Arkansas at Little Rock \\ 2801 S. University Avenue \\ Little Rock, AR 72204 \\ lmdavis@ualr.edu
}

\author{
Ajit Kambil \\ CFO Program \\ Deloitte LLP \\ 1633 Broadway \\ New York, NY 10019 USA \\ akambil@deloitte.com
}

This minitrack continues in its sixteenth year, highlighting some of the most interesting studies in this area. We have accepted papers in two areas: 1) quantitative, empirical research with strong theoretical underpinnings, and 2) novel methods and approaches, including case studies and frameworks, for envisioning and creating effective forms of online marketing.

In "Is the Whole Equal to the Sum of its Parts? Exploring the Impact of Inconsistency on Perceived Helpfulness of a Set of Reviews," Dezhi Yin, Triparna de Vreede, Logan Macray Steele, and Gert-Jan de Vreede employ bounded rationality theories to assess the usefulness in a set of reviews. Using an experimental approach, they find that the whole differs from the sum of its parts when reviews are consistent with each other or are contradicting each other, but does not differ when the reviews contain mixed, non-contradicting opinions.

Tommi Mahlamaki, Kati Stormi, Lauri Saarivuori, and Mika Ojala, in "Electronic Marketing in Business-toBusiness Markets - User Related Benefits of Sales Configurators," focus on categorizing and identifying the most important benefits of sales configurators for B2B markets. User experience, and security were found to be most important; other important factors are versatility, configurability, and customizability.

Yongqiang Sun, Yanping Guo, Dina Liu, and Nan Wang, in 'Exploring Consumers' Continuance Intention to Use Peer-to-Peer Accommodation Service: The Role of Psychological Ownership," study customer retention in peer-to-peer accommodation services. They find that novelty-seeking, home benefits and social interaction have significant effects on psychological ownership, which, in turn, can, through affective commitment, influence consumers' intention to purchase again.

In "Cross-Channel Integration and Customer Retention in Omnichannel Retailing: The Role of Retailer Image and Alternative Attractiveness," Ruizhi Li, Yang Li, Hefu Liu, and Qian Huang explore the contingency role of retailer image and alternative attractiveness on the relationship between cross-channel integration and customer retention. They find a positive relationship, and that retailer image and alternative attractiveness moderate the relationship.
Ling Liu, and Aoxuan Liu, in "Intrinsic Characteristics or Earned It? A Discussion of Firm Attractive Traits and Their Social Media Engagement," study firm use of social media for attracting users. They find that there are "engaging traits" that attract users, and that firms "born-to-be" less attractive, can employ social media engagements to increase their popularity levels and to eventually enjoy improved marketing and management performance.

In "How Mobile Applications Affect Consumption in Grocery Stores," Anne Kokkonen, and Tommi Laukkanen examine how utilitarian and hedonic values drive customer recommendation behavior and engagement with mobile grocery shopping application. Findings show that usage frequency of the mobile application increases money spent on a retail chain in all consumer segments except Millennials, and that there are gender and generational differences in the role perceived value plays in customers' recommendation behavior and engagement with mobile grocery shopping applications.

In "Management Response to Online Customer Reviews in Distribution Channel: A Perspective of Manufacturers," Di Li, Ruhai Wu, and Hu Wang investigate the relationship between a manufacturers' management response strategies to online customer reviews and the channel structure formed in the online market. Based on data collected from Amazon.com, they find that if a manufacturer invests in responding to customer reviews, then more retailers are willing to carry its product(s); and if more retailers sell a manufacturer's product(s), or if there is more intensive intra-competition in the retailer channels, the manufacturer is more likely to invest in management response.

Eugenia Y. Huang, Sheng-Wei Lin, and Kai-Teng Cheng, in "How does Omnichannel Integration Quality Affect Consumers' Stickiness Intention," Use commitment-trust theory to examine the effects of omnichannel integration quality on consumers' stickiness intention. They find that show that high levels of omnichannel integration quality can increase consumer trust, which helps retailers maintain favorable customer relationships; and that consumer trust and relationship commitment have a strong influence on stickiness intention, and breadth, transparency, and process consistency directly affect trust. 